\title{
Lysol Lakes, and Other Photographs
}

\section{Brittany Schaefer}

\begin{abstract}
Since the worldwide implementation of lockdown measures due to COVID-19, there have been substantial changes in how we interact with our social and material worlds. Urban landscapes are being reimagined (Pierantoni et al., 2020), individuals are finding new ways to re-create outdoors (Rice et al., 2020), and many health services are being digitized to increase accessibility (Taylor et al., 2020). This short photo essay adds a visual component to such everyday social and material changes. More specifically, it is composed of three photographs that I took while on my solo-walks in Southern Ontario during the initial lockdown. The collection explores three themes: the irony of cleanliness, danger and contamination, and the importance of staying connected with loved ones during COVID-19.
\end{abstract}

\section{Keywords}

COVID-19, materiality, contamination, sociality, lockdown 


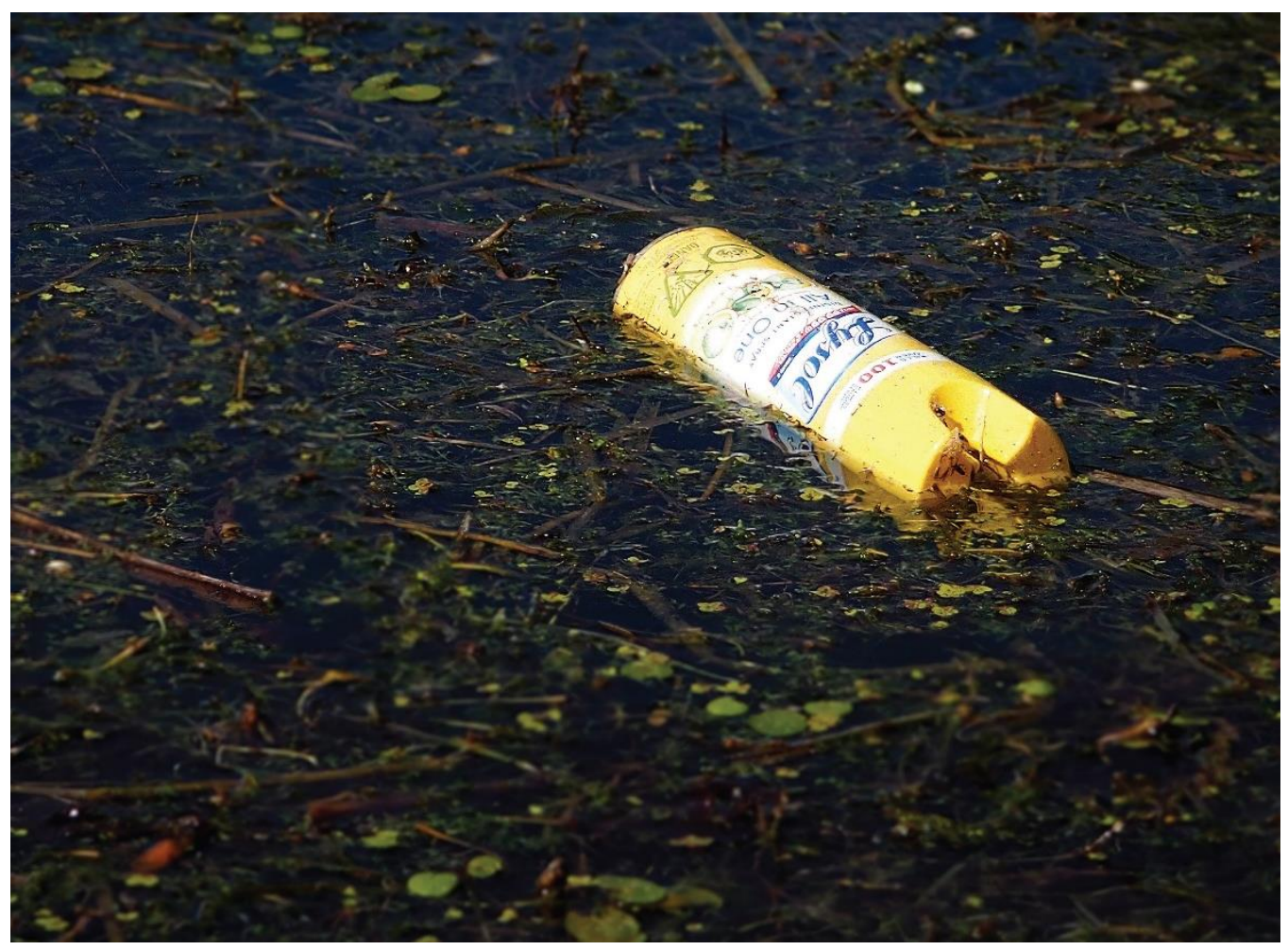

\section{Lysol Lakes}

Among the aquatic vegetation and other flotsam occupying the harbour, you can see the bright yellow beacon of a discarded disinfectant aerosol canister. There is an irony here somewhere; the newfound obsession with cleanliness is simultaneously contaminating our lakes. Increased consumption of disinfectant products is another part of our changing material reality. 


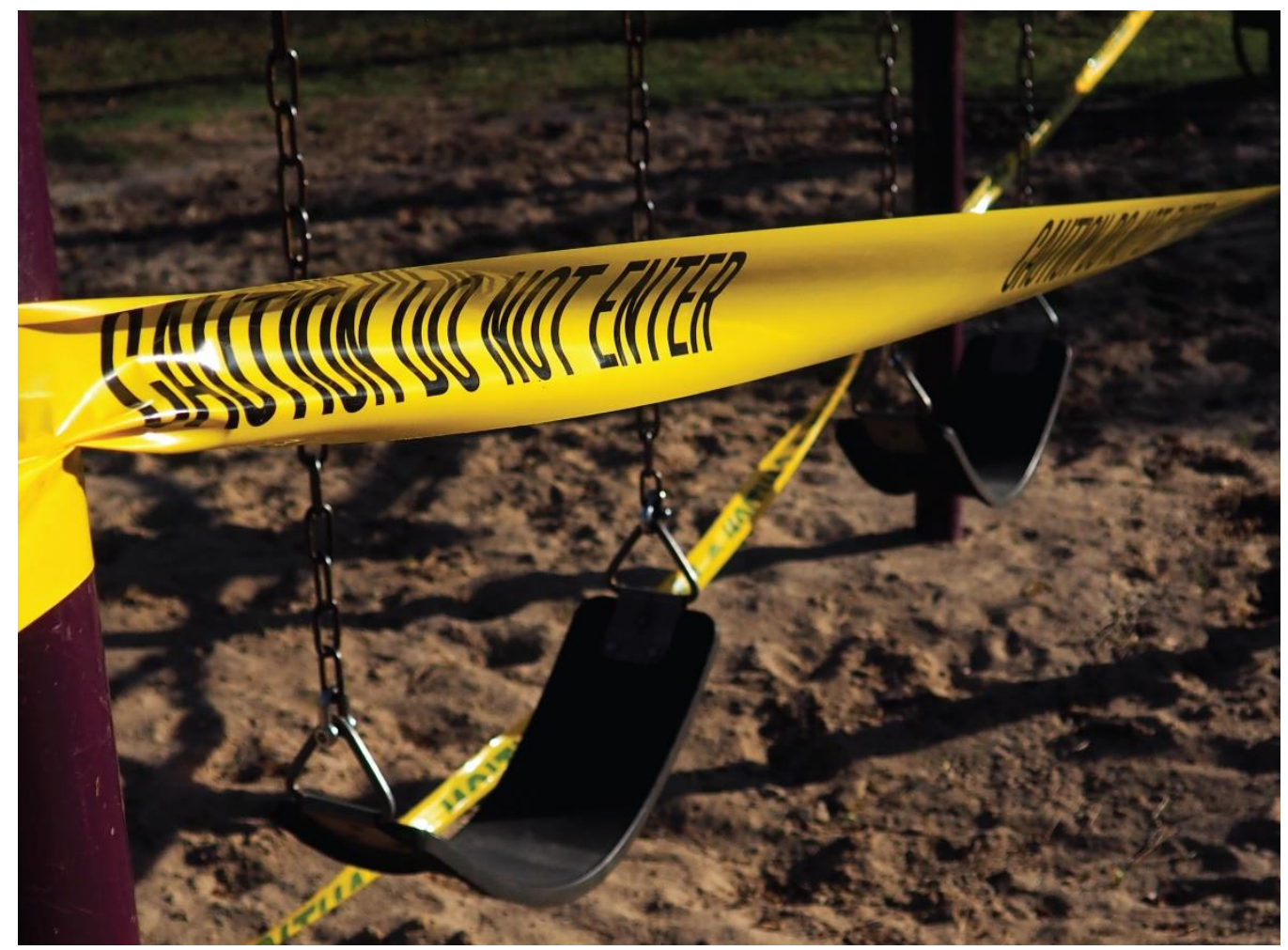

\section{No Fun Allowed}

No longer a place of climbing, laughter, games, and scraped knees, this playground became a site of danger and contamination. Caution, it says, Do Not Enter. The ordinary is now a threat. The COVID-19 pandemic has imposed constraints on our ability to exist outside, and the repercussions of this will disproportionately affect those who rely on these resources the most. 


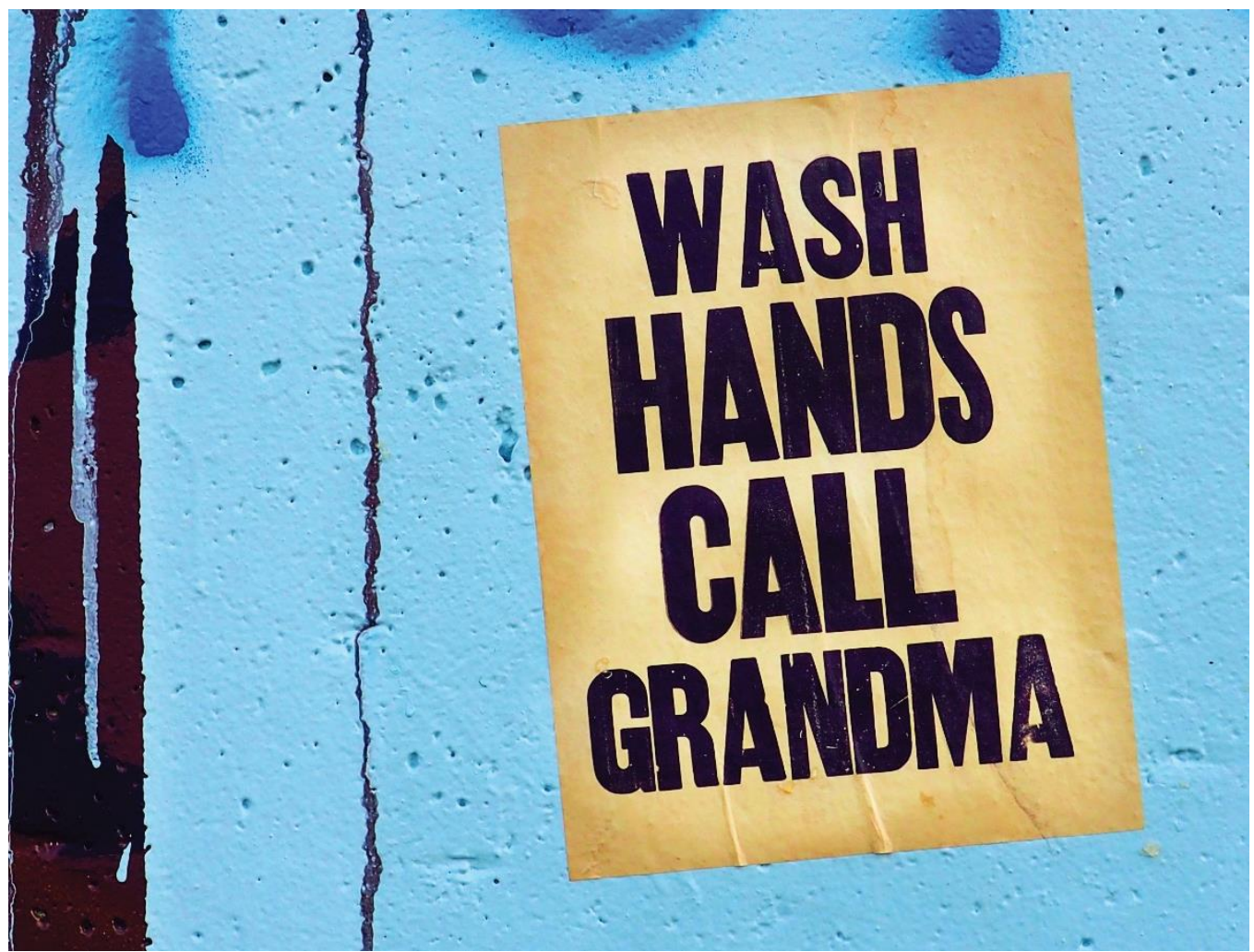

\section{Wash Hands, Call Grandma}

Aside from my father dropping off groceries, my grandmother was alone for the first weeks of the provincial stay-home advisory policy. Despite her simple request for a visit from her grandchildren, we did not feel comfortable visiting. What if we gave her COVID? She insisted that we come see her. She said that she would rather have a shorter life surrounded by family than extend it by a few lonely years.

This photo serves as both a reminder for maintaining proper sanitary hygiene, and of the importance of reaching out to our loved ones.

Wash your hands. Wear a mask. Call Grandma. Stay safe. 


\section{References}

Pierantoni, I., Pierantozzi, M., and Sargolini, M. (2020). COVID 19--A qualitative review for the reorganization of human living environments. Applied Sciences, 10(16), 5576.

Taylor, C. B., Fitzsimmons-Craft, E. E., and Graham, A. K. (2020). Digital technology can revolutionize mental health services delivery: The COVID-19 crisis as a catalyst for change. The International Journal of Eating Disorders, 53(7), 1155-1157.

Rice, W. L., Mateer, T. Taff, B. D., Lawhon, B, Reigner, N., and Newman, P. (2020). The COVID-19 pandemic continues to change the way people recreate outdoors. Leave No Trace Center for Outdoor Ethics. https://doi. org/10.31235/osf.io/dghba

\section{Author biography}

Brittany Schaefer is an MA student in Public Issues Anthropology at the University of Guelph. Her research interests sit between the disciplines of environmental anthropology, gender, and leisure and tourism studies. Pre-COVID, she had prepared to collect data for her thesis project on tourism and waste in coastal communities. The project was titled, Living with Waste and Caring for Place: Trash and Tourism in Koh Lanta, Thailand. This research is on hold until further notice. Dismayed by the unprecedented pressures of the "new normal", Brittany began taking photographs on her walks around the neighbourhood. Being able to see the world through the camera's lens brought a new perspective and has helped her to focus on the positives in her new position as a person living through a global health pandemic. Currently, Brittany is preparing a new thesis project focused on how humans interact with the nonhuman worlds in Ontario. Specifically, she will be exploring how rock climbers engage in reciprocal relationships with the more-than-human world of the Niagara Escarpment with attention to the material aspects of conservation and care. When she is not reading, grading, or climbing, she can be found trying to cultivate a reciprocal relationship with her houseplants. So far, she has had limited success. 